\title{
RATA: Rapid Atomic Type Analysis by Abstract Interpretation - Application to JavaScript Optimization
}

\author{
Francesco Logozzo and Herman Venter \\ Microsoft Research, Redmond, WA (USA) \\ \{logozzo, hermanv\}@microsoft.com
}

\begin{abstract}
We introduce RATA, a static analysis based on abstract interpretation for the rapid inference of atomic types in JavaScript programs. RATA enables aggressive type specialization optimizations in dynamic languages. RATA is a combination of an interval analysis (to determine the range of variables), a kind analysis (to determine if a variable may assume fractional values, or $\mathrm{NaN}$ ), and a variation analysis (to relate the values of variables). The combination of those three analyses allows our compiler to specialize Float64 variables (the only numerical type in JavaScript) to Int32 variables, providing large performance improvements (up to $7.7 \times$ ) in some of our benchmarks.
\end{abstract}

\section{Introduction}

JavaScript is probably the most widespread programming platform in the world. JavaScript is an object-oriented, dynamically typed language with closures and higher-order functions. JavaScript runtimes can be found in every WEB browser (e.g., Internet Explorer,Firefox, Safari and so on) and in popular software such as Adobe Acrobat and Adobe Flash. Large and complex WEB applications such as Microsoft Office WEB Apps or Google Mail, rely on JavaScript to run inside every browser on the planet.

A fast JavaScript implementation is crucial to provide a good user experience for rich WEB applications and hence enabling their success. Because of its dynamic nature, a JavaScript program cannot statically be compiled to efficient machine code. A fully interpreted solution for JavaScript runtime is generally acknowledged to be too slow for the new generation of web applications. Modern implementations rely on Just-in-time (JIT) techniques: When a function $f$ is invoked at runtime, $f$ is compiled to a function $f^{\prime}$ in machine code, and it is then executed. The performance gain of executing $f^{\prime}$ pays off the extra time spent in the compilation of $f$. The quality of the code that the JIT generates for $f^{\prime}$ depends on the amount of dynamic and static information that is available to it at the moment of the invocation of $f$. For instance, if the JIT knows that a certain variable is of an atomic type then it generates specialized machine instructions (e.g., incr for an Int32) instead of relying on expensive boxing/unboxing operations. 
Motivating Example. Let us consider the nestedLoops function in Fig. 1] Without any knowledge of the concrete types of $i$ and $j$, the JIT should generate a value wrapper containing: (i) a tag with the dynamic type of the value, and (ii) the value. Value wrappers are disastrous for performance. For instance, the execution of nestedLoops takes $310 \mathrm{~ms}$ on our laptop. 1 In fact, the dynamic execution of the statement $i++$ involves: (i) an "unbox" operation to fetch the old value of $i$ and check that it is a numerical type; (ii) incrementing $i$; (iii) a "box" operation to update the wrapper with the new value. The JIT can specialize the function if it knows that $i$ and $j$ are numerical values. In JavaScript, the only numerical type is a 64 bits floating point (Float64) which follows the IEEE754 standard [1619]. In our case, a simple type inference can determine that $i$ and $\mathrm{j}$ are Float64: they are initialized to zero and only incremented by one. The execution time then goes down to $180 \mathrm{~ms}$.

The JIT may do a better job if it knows that $i$ and $j$ are Int32: floating point comparisons are quite inefficient and they usually requires twice or more instructions to perform than integer comparisons on a x86 architecture. A simple type inference does not help, as it cannot infer that $i$ and $j$ are bounded by 10000. In fact, it is safe to specialize a numerical variable $\mathrm{x}$ with type Int32 when one can prove that for all possible executions:

(i) $\mathrm{x}$ never assumes values outside of the range $\left[-2^{31}, 2^{31}-1\right]$; and

(ii) $\mathrm{x}$ is never assigned a fractional value $(e . g ., 0.5)$.

Contribution. We introduce RATA, Rapid Atomic Type Analysis, a new static analysis based on abstract interpretation, to quickly and precisely infer the numerical types of variables. RATA is based on a combination of an interval analysis (to determine the range of variables), a kind analysis (to determine if a variable may assume fractional values, or $\mathrm{NaN}$ ) and a variation analysis (to relate the values of variables). In our example, the first analysis discovers that $i \in[0,10000], j \in[0,10000]$ and the second that $i, j \in \mathbb{Z}$. Using this information, the JIT can further specialize the code so that $i$ and $j$ are allocated in integer registers, and as a matter of fact the execution time (inclusive of the analysis time) drops to $31 \mathrm{~ms}$ !

The function bitsinbyte in Fig. 1 (extracted from the SunSpider benchmarks [31]) illustrates the need for the variation analysis. The interval analysis determines that $\mathrm{m} \in[1,256], \mathrm{c} \in[0,+\infty]$. The kind analysis determines that $\mathrm{m}, \mathrm{c} \in \mathbb{Z}$. If we infer that $\mathrm{c} \leq \mathrm{m}$ then we can conclude that $\mathrm{c}$ is an Int32. In general, we can solve this problem using a relational, or weakly relational abstract domain, such as Polyhedra [12, Subpolyhedra [23], Octagons [26], or Pentagons 24. However, all those abstract domains have a cost which is quadratic (Pentagons), cubic (Octagons), polynomial (Subpolyhedra) or exponential (Polyhedra) and hence we rejected their use, as non-linear costs are simply not tolerable at runtime. Our variation analysis infers that: (i) $\mathrm{m}$ and c differ by one

${ }^{1}$ The data we report is based on the experience with our own implementation of a JavaScript interpreter for .Net. More details will be given in Sect. 6. 


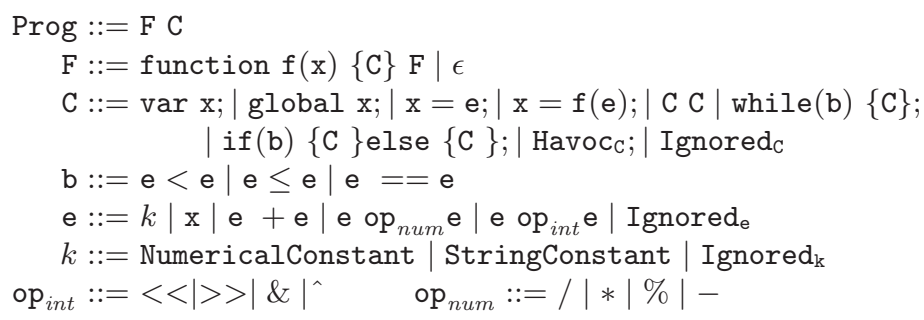

Fig. 2. The syntax of the JavaScript $=$ language

combined with binary operators. We distinguish three kinds of binary operators: (i) sum, +, which can be either the usual IEEE754 addition when its operands are numerical values or string concatenation otherwise; (ii) numerical operations which return a numerical value (or $\mathrm{NaN}$ if the operation is undefined, e.g. 0/0); (iii) int operations, which always return a Int32 value. The expression Ignored abstracts the expressions that we do not consider here such as Boolean operators and casting. A constant can either be an IEEE754 64-bits numerical constant, a string literal or some constant we do not deal with (e.g., Boolean constants).

It is worth mentioning that even if in the definition of JavaScript $=$ we ignore some language constructs, our implementation takes care of them e.g. by syntax rewriting ( $\mathrm{x}+=2$ " $\rightarrow$ " $\mathrm{x}=\mathrm{x}+2$ ").

\section{Background}

\subsection{IEEE754 Standard}

The IEEE754 standard defines, among other things, the arithmetic format for floating point computations. When using 64-bits (Float64), the standard format allows numbers as large as $\pm 1.7976931348623157 \cdot 10^{308}$ and as small as $\pm 5 \cdot 10^{-324}$ to be represented. All the integers between $-2^{53}$ and $2^{53}$ are exactly represented. Outside of this interval, one may lose precision in the trailing digits. Unlike machine integers: (i) Float64 numbers do not overflow, and (ii) two special values represent infinities: $\pm \infty$. For instance, $1 / 0=+\infty=+\infty+10$. The Float64 format also specifies a special value NaN (Not-a-Number) as the result of invalid operations, e.g.,$\infty / \infty$. A peculiar property of $\mathrm{NaN}$ is that $\mathrm{NaN} \neq \mathrm{NaN}$.

One can specialize a Float64 variable $\mathrm{x}$ to a Int32 without changing the semantics of the program if one can prove that $\mathrm{x}$ will never assume: (i) a fractional value, a NaN or an infinity; and (ii) a value outside of the range $\left[-2^{31}, 2^{31}-1\right]$. The goal of RATA is to enable such specialization.

\subsection{Abstract Interpretation}

Abstract interpretation [1011] is a general theory of semantic approximations. Its more interesting application is to define and prove soundness of program analyses. From the abstract interpretation perspective, a static analysis is a program 
semantics that is coarse enough to be computable and precise enough to capture the properties of interests. The concrete semantics of a program is defined over a complete lattice $\langle C, \sqsubseteq\rangle$. The abstract semantics is defined as a fixpoint over a complete lattice $\langle\mathrm{A}, \underline{\sqsubseteq}\rangle$, which is related to $\mathrm{C}$ by a Galois connection, i.e., a pair of monotonic functions $\langle\alpha, \gamma\rangle$ such that $\forall \mathrm{c} \in \mathrm{C}$. c $\sqsubseteq \gamma \circ \alpha(\mathrm{c})$ and $\forall \overline{\mathrm{a}} \in \mathrm{A} . \alpha \circ \gamma(\overline{\mathrm{a}}) \overline{\bar{a}}$. We write $\langle\mathrm{C}, \sqsubseteq\rangle \stackrel{\gamma}{\leftrightarrows}\langle\mathrm{A}, \sqsubseteq \bar{\zeta}\rangle$ to denote that. An abstract transfer function $\bar{\tau}$ is a sound approximation of a concrete $\tau$ if $\forall \bar{a} \in A$. $\alpha \circ \tau \circ \gamma(\bar{a}) \sqsubseteq \bar{\tau}(\bar{a})$. In general, the abstract domain A may contain strictly increasing infinite (or very long) chain. Hence, to ensure the convergence of fixpoint iterations one should use a widening operator, which extrapolates the limit of the sequence. Precision lost by the widening can be recovered using a narrowing operator.

\section{Numerical Abstract Domains}

The Rapid Atomic Type Analysis (RATA) is meant to be used in an online context, as an oracle for the JIT that can use the inferred types to generate more specialized code. RATA is a combination of three different static analyses. An interval analysis to determine the range of the variables. A kind analysis to infer if a variable can assume a fractional or a NaN value. A variation analysis to infer loose relationships about program variables, and hence refine the ranges and the kinds. The analysis should be very fast, to avoid causing untoward pauses in normal program execution. We rejected the use of expressive yet expensive numerical abstract domains. For instance, Octagons have a cubic complexity (in the number of program variables), Polyhedra are exponential, and Subpolyhedra lay in between.

\subsection{Extended Intervals}

The interval abstract domain was introduced by Cousot \& Cousot in 10] as example of the application of Abstract Interpretation to program optimization (specifically array bounds check removal). Inspired by this idea, we use it for type specialization. Our extended intervals are a little bit different from the originals, in that we also consider intervals potentially containing NaN, intervals abstracting non-numerical values, intervals abstracting floats and intervals bounded only by Int 32 values. An interval can either be the empty interval, the interval containing only NaN, a Int32-bounded interval, an open interval or the unknown interval $\left(\top_{i}\right)$ :

$$
\begin{gathered}
\operatorname{Intv}=\perp_{i}|\operatorname{NaN}| \operatorname{Normal}(a, b) \mid \text { OpenLeft }(b) \mid \text { OpenRight }(a) \mid \top_{i} \\
a, b \in \operatorname{Int32}
\end{gathered}
$$

More formally, the meaning of an interval is given by the concretization function $\gamma_{i} \in[\operatorname{lntv} \rightarrow \mathcal{P}(\mathrm{Val})]$. The set $\mathrm{Val}$ is the set of concrete JavaScript $=$ values. We are interested only in numerical values, so we let $V_{a l}=\operatorname{lgnored}_{V_{a}} \cup \mathbb{R} \cup\{ \pm \infty, N a N\}$. For simplicity, we let $\mathbb{R}^{*}=\mathbb{R} \cup\{ \pm \infty, \mathrm{NaN}\}$, and we extend the usual axioms over reals so that $\forall r \in \mathbb{R}$. $-\infty<r<+\infty$ and $\forall r \in \mathbb{R}^{*} . r \neq$ NaN. The concretization function and the induced order $\sqsubseteq_{i}$ are in Fig. 3 . 

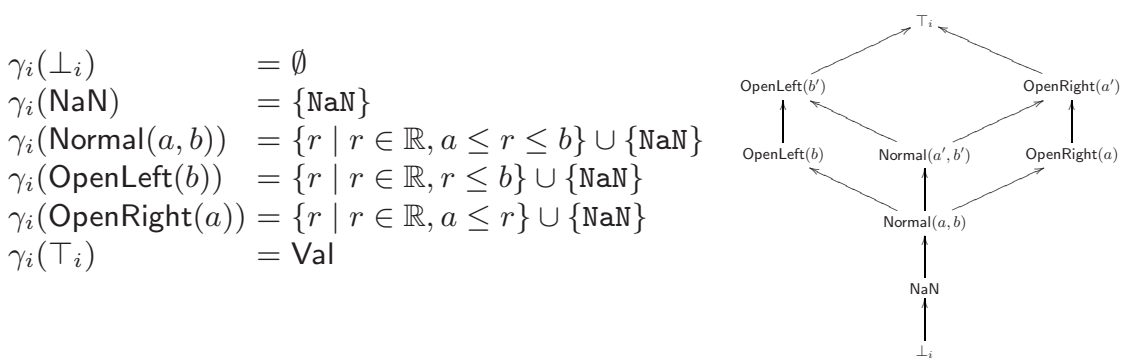

Fig. 3. The concretization $\gamma_{i}$ and the order $\sqsubseteq_{i}$ on the extended intervals. We assume that $a^{\prime} \leq a \leq b \leq b^{\prime}$.

Example 1. $\gamma_{i}($ OpenRight $(10))=\{10 \ldots 11 \cdots+\infty\} \cup\{\mathrm{NaN}\}$.

The abstraction function $\alpha_{i} \in[\mathcal{P}(\mathrm{Val}) \rightarrow \operatorname{lntv}]$ is defined as

$$
\begin{aligned}
& \alpha_{i}(R)= \bigsqcup_{i}\left\{\dot{\alpha}_{i}(r) \mid r \in R\right\} \text { where } \\
& \dot{\alpha}_{i}(r)= \begin{cases}\text { NaN } & r \text { is NaN } \\
\text { OpenRight }\left(2^{31}-1\right) & 2^{31}-1<r \leq+\infty \\
\text { OpenLeft }\left(-2^{31}\right) & -\infty \leq r<-2^{31} \\
\text { Normal }(\text { floor }(r), \operatorname{ceiling}(r)) & -2^{31} \leq r \leq 2^{31}-1 \\
\top_{i} & \text { otherwise }\end{cases}
\end{aligned}
$$

(floor $(r)=\max \{x \in \mathbb{Z} \mid x \leq r\}$ and ceiling $(r)=\min \{x \in \mathbb{Z} \mid r \leq x\}$ ).

Example 2. $\alpha_{i}(\{10.3,+\infty, \mathrm{NaN}\})=$ OpenRight $(10), \alpha_{i}(\{3.14\})=\operatorname{Normal}(3,4)$.

Theorem 1. $\langle\mathcal{P}(\mathrm{Val}), \subseteq\rangle \underset{\alpha_{i}}{\leftrightarrows}\left\langle\operatorname{lntv}, \sqsubseteq_{i}\right\rangle$.

It is worth noting that for Th. 1 to hold we need to map $\pm \infty$ to the smallest abstract element containing $\pm \infty$.

The abstract domain Intv is precise enough to capture that the value of a variable is always within the Int32 range, but it cannot capture the fact that a variable never assumes fractional values, crucial for soundness : e.g., $1 / 2$ is 0.5 with Float64 semantics and 0 with Int32 semantics.

Example 3. For the function bitsinbyte, the analysis with Intv infers that $\mathrm{m}$ : Normal(0,512), c : OpenRight(0), b : $\top_{i}$.

\subsection{Kinds}

The elements of the Kind abstract domain are either the empty kind, a 32-bits integer, a 64-bit floating point number or an unknown kind of value:

$$
\text { Kind }=\perp_{k} \mid \text { Int32 } \mid \text { Float64 } \mid \top_{k} .
$$


$\begin{array}{ll}\gamma_{k}\left(\perp_{k}\right) & =\emptyset \\ \gamma_{i}(\text { Int32 }) & =\left\{r \mid r \in \mathbb{Z},-2^{31} \leq r \leq 2^{31}-1\right\} \\ \gamma_{i}(\text { Float64 }) & =\left\{r \mid r \in \mathbb{R}^{*}, r \text { is a } 64 \text { bits IEEE } 754 \text { number }\right\} \\ \gamma_{i}\left(\top_{k}\right) & =\text { Val }\end{array}$

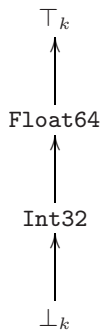

Fig. 4. The concretization $\gamma_{k}$ and the order $\sqsubseteq_{k}$ of the Kinds

The meaning function $\gamma_{k} \in[$ Kind $\rightarrow \mathcal{P}(\mathrm{Val})]$ and the induced order $\sqsubseteq_{k}$ are in Fig. 4. The abstraction function $\alpha_{k} \in[\mathcal{P}($ Val $) \rightarrow$ Kind $]$ is

$$
\alpha_{k}(R)=\bigsqcup_{k}\left\{\dot{\alpha_{k}}(r) \mid r \in R\right\} \quad \text { where } \dot{\alpha_{k}}(r)= \begin{cases}\text { Int32 } & r \text { is a Int32 } \\ \text { Float64 } & r \text { is a Float64 } \\ \top_{k} & r \text { otherwise }\end{cases}
$$

Example 4. $\alpha_{k}(\{10.3,+\infty, \mathrm{NaN}\})=\alpha_{k}(\{3.14\})=$ Float 64.

Theorem 2. $\langle\mathcal{P}(\mathrm{Val}), \subseteq\rangle \underset{\alpha_{k}}{\stackrel{\gamma_{k}}{\leftrightarrows}}\left\langle\right.$ Kind, $\left.\sqsubseteq_{k}\right\rangle$.

The abstract domain of Kind in isolation is of almost no use (maybe except for trivial, loop free programs). In the nestedloops example, knowing that $i$ is initialized to a Int32, it is compared to a Int32, and only incremented by one it is not enough to deduce that $i$ is an Int32. In fact if the loop guard were instead $i \leq 2^{31}$, then after the last iteration of the loop $i=2^{31}+1$ which is a fine Float64 value, but not an Int32.

\subsection{K-Intervals}

The combination of extended intervals and kinds allow the derivation of very powerful yet rapid analyses. We call the reduced product of Kind and Intv a $\mathrm{k}$-interval. The elements of the abstract domain are pairs in Intv $\times$ Kind, and the concretization $\gamma_{k i} \in[\operatorname{Intv} \times \mathrm{Kind} \rightarrow \mathcal{P}(\mathrm{Val})]$ is $\gamma_{k i}(\langle i, k\rangle)=\gamma_{i}(i) \cap \gamma_{k}(k)$. The abstraction $\alpha_{k i} \in[\mathcal{P}(\mathrm{Val}) \rightarrow \operatorname{Intv} \times$ Kind $]$ is the simple pairwise abstraction: $\alpha_{k i}(R)=\left\langle\alpha_{i}(R), \alpha_{k}(R)\right\rangle$. The order $\sqsubseteq_{k i}$ is the pairwise extension of the order on the basic domains. We write $\mathrm{x}: t$ to denote that the variable $\mathrm{x}$ has a k-interval $t$.

Theorem 3. $\langle\mathcal{P}(\mathrm{Val}), \subseteq\rangle \underset{\alpha_{k i}}{\stackrel{\gamma_{k i}}{\leftrightarrows}}\left\langle\operatorname{lntv} \times\right.$ Kind, $\left.\sqsubseteq_{k i}\right\rangle$

K-Intervals are more expressive than the single domains and can represent addition information, crucial to type specialization: 


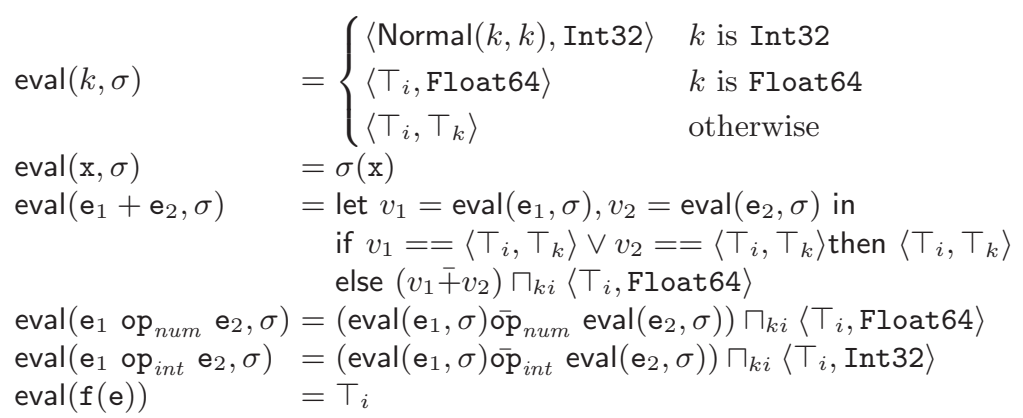

Fig. 6. The abstract evaluation of expressions. The abstract operators $\overline{+}, \overline{\mathrm{op}}_{\text {num }}, \overline{\mathrm{p}}_{\text {int }}$ are the abstract counterparts of concrete the concrete operators.

Initial State. At the entry point of $f$, the global values are set to $\left\langle\top_{i}, \top_{k}\right\rangle$ (any value), the local values are set to $\left\langle\perp_{i}, \perp_{k}\right\rangle$ (uninitialized), and the actual value $v$ of the parameter $\mathrm{x}$ is generalized to $\left\langle\top_{i}, \alpha_{k}(\{v\})\right\rangle$. We generalize the actual value of the parameter so that the result of the analyses can be re-used.

Example 6. The initial abstract state for the analysis of 1oopToN(99999) in Fig.5 is $\sigma_{0}=\left[\mathrm{n} \mapsto\left\langle T_{i}, \operatorname{Int} 32\right\rangle, \mathrm{x} \mapsto\left\langle\perp_{i}, \perp_{k}\right\rangle\right]$. The specialization of loopToN can be cached and reused for loopToN(1234) as $\sigma_{0}$ is an over-approximation of $[\mathrm{n} \mapsto$ $\left.\langle\operatorname{Normal}(1234,1234), \operatorname{Int} 32\rangle, \mathrm{x} \mapsto\left\langle\perp_{i}, \perp_{k}\right\rangle\right]$.

Variables. RATA is a modular analysis, run on a per-method basis. In the general case, at the moment of the invocation of RATA, we have not seen all the assignments to globals, so that the only sound assumption for globals is the open-world assumption, i.e., they can assume any value.

Assignment. An assignment $\mathrm{x}=\mathrm{e}$ in a pre-state $\sigma_{0}$, updates the entry for $\mathrm{x}$ with eval $\left(\mathrm{e}, \sigma_{0}\right)$ if $\mathrm{x}$ is a local variable (or a parameter) or it does nothing otherwise. The evaluation function eval $\in[\mathrm{e} \times \Sigma \rightarrow$ Intv $\times$ Kind $]$ is in Fig. 6. The k-interval for a constant is assigned according to its type. The "+" operator is polymorphic in JavaScript: it can either be string concatenation or numerical addition. As a consequence, if no information on the operands is available, nothing can be inferred on the result. Otherwise, we know that it is at least a Float64. The result of a op $\mathrm{p}_{\text {num }}\left(\mathrm{op}_{\text {int }}\right)$ is at least a Float64 (an Int32). The return value of a function call is ignored: to statically determine which function is invoked requires a quite complex global program analysis, out of the scope of this paper.

Test. A precise handling of tests enables the refinement of the abstract states, and hence a more precise analysis. A too precise analysis of tests (e.g., using forward/backwards iterations [9]) may cause slowdowns unacceptable for an online analysis. In our implementation we only consider comparisons between a variable and an expression, or between two variables. For equalities, we have that:

$$
\begin{aligned}
& \mathbb{N} \llbracket \mathrm{x}==\mathrm{e} \rrbracket\left(\sigma_{0}\right)=\sigma_{0}\left[\mathrm{x} \mapsto \sigma_{0}(\mathrm{x}) \sqcap_{k i} \text { eval }\left(\mathrm{e}, \sigma_{0}\right)\right], \text { and } \\
& \mathbb{N} \llbracket \mathrm{x}==\mathrm{y} \rrbracket\left(\sigma_{0}\right)=\sigma_{0}\left[\mathrm{x}, \mathrm{y} \mapsto \sigma_{0}(\mathrm{x}) \sqcap_{k i} \sigma_{0}(\mathrm{y})\right] .
\end{aligned}
$$


For an inequality $\mathrm{x}<\mathrm{e}$, the upper bound of $\mathrm{x}$ is refined by the upper bound of eval $\left(\mathrm{e}, \sigma_{0}\right)(\operatorname{upp}(\langle i, t\rangle)$ is the open k-interval bounded by the upper bound of $i$, it can be $+\infty)$ :

$$
\mathbb{N} \llbracket \mathrm{x}<\mathrm{e} \rrbracket\left(\sigma_{0}\right)=\sigma_{0}\left[\mathrm{x} \mapsto \sigma_{0}(\mathrm{x}) \sqcap_{k i} \operatorname{upp}\left(\operatorname{eval}\left(\mathrm{e}, \sigma_{0}\right)\right)\right] .
$$

Similarly for an inequality $\mathrm{x}<\mathrm{y}$, the upper bound for $\mathrm{x}$ can be refined by the upper bound of $y$, and the lower bound of $y$ can be refined by the lower bound of $\mathrm{x}$ :

$\mathbb{N} \llbracket \mathrm{x}<\mathrm{y} \rrbracket\left(\sigma_{0}\right)=\sigma_{0}\left[\mathrm{x} \mapsto \sigma_{0}(\mathrm{x}) \sqcap_{k i} \operatorname{upp}\left(\operatorname{eval}\left(\mathrm{y}, \sigma_{0}\right)\right), \mathrm{y} \mapsto \sigma_{0}(\mathrm{y}) \sqcap_{k i} \operatorname{low}\left(\operatorname{eval}\left(\mathrm{x}, \sigma_{0}\right)\right)\right]$.

Example 7. Let us assume that $\sigma_{0}=\left[\mathrm{x} \mapsto\left\langle\right.\right.$ OpenRight $\left.\left.(10), \top_{i}\right\rangle\right]$. Then:

$$
\mathbb{N} \llbracket \mathrm{x}<1000 \rrbracket\left(\sigma_{0}\right)=\left[\mathrm{x} \mapsto\left\langle\operatorname{Normal}(10,1000), \top_{i}\right\rangle\right] .
$$

Note that it would be unsound to assume that $\mathrm{x}$ is an Int32 or that $\mathrm{x}$ : Normal(10, 999).

Sequence. The analysis of a sequence of statements is the composition of the analyses: $\mathbb{N} \llbracket \mathbf{C}_{1} \mathbf{C}_{2} \rrbracket\left(\sigma_{0}\right)=\mathbb{N} \llbracket \mathbf{C}_{2} \rrbracket\left(\mathbb{N} \llbracket \mathbf{C}_{1} \rrbracket\left(\sigma_{0}\right)\right)$.

Conditional. For a conditional the analysis first refines the pre-state with the guards, and then joins the results (the function Not negates the Boolean expression $\mathrm{b})$ :

$$
\mathbb{N} \llbracket \text { if }(\mathrm{b})\left\{\mathrm{C}_{1}\right\} \text { else }\left\{\mathrm{C}_{2}\right\} ; \rrbracket\left(\sigma_{0}\right)=\mathbb{N} \llbracket \mathrm{C}_{1} \rrbracket\left(\mathbb{N} \llbracket \mathrm{b} \rrbracket\left(\sigma_{0}\right)\right) \sqcup_{k i} \mathbb{N} \llbracket \mathrm{C}_{2} \rrbracket\left(\mathbb{N} \llbracket \operatorname{Not}(\mathrm{b}) \rrbracket\left(\sigma_{0}\right)\right) .
$$

Loop. A loop invariant for while(b) $\{\mathrm{C}\}$; is a fixpoint of the functional $F \in$ $[\Sigma \rightarrow \Sigma]:$

$$
F(X)=\sigma_{0} \dot{ப}_{k i} \mathbb{N} \llbracket C \rrbracket(\mathbb{N} \llbracket \mathrm{b} \rrbracket(X)),
$$

where $\sigma_{0}$ is the abstract state at the entry point of the loop and $\dot{ப}_{k i}$ is the pointwise extension of $\sqcup_{k i}$. An invariant can be computed with the usual fixpoint iteration techniques. The abstract domain Intv $\times$ Kind does not contain infinite ascending chains, but it contains very very long chains (up to $2^{32}+3$ elements). We need a widening operator to speed up the convergence of the iterations to a post-fixpoint. A widening with thresholds 622, and the re-execution from a post-fixpoint (a form of narrowing [22]) guarantee a good precision yet providing good performance. We illustrate those two techniques with examples.

Example 8. The iterations with the classical widening for the loop function of Fig. 5 produce the following sequence of abstract values for $\mathrm{x}$ :

$$
\langle\operatorname{Normal}(0,0), \operatorname{Int} 32\rangle \sqsubseteq_{k i}\langle\operatorname{Normal}(0,1), \operatorname{Int32}\rangle \sqsubseteq_{k i}\langle\text { OpenRight }(0), \text { Float64 }\rangle,
$$

as the upper bound for $\mathrm{x}$ is extrapolated to $+\infty$. The threshold (or staged) widening tries to extrapolate the upper bound to constants appearing in guards, producing the sequence:

$\langle\operatorname{Normal}(0,0), \operatorname{Int32}\rangle \sqsubseteq_{k i}\langle\operatorname{Normal}(0,1), \operatorname{Int32}\rangle \sqsubseteq_{k i}\langle\operatorname{Normal}(0,10000), \operatorname{Int} 32\rangle$. 
In general, during the analysis we collect all the constants that appear in the tests, and we use them as steps for widening with a threshold.

Example 9. The type of $\mathrm{x}$ in function loopToN of Fig. 5 depends on the input parameter. When it is invoked with an Int32 value, then we would like RATA to discover that $\mathrm{x}$ is an Int32. Widening with thresholds is of no help here (there are no constants in guards) so the iterations stabilize at $I=\langle$ OpenRight (0), Float64 . A re-execution of the loop with initial state $I$ will refine the abstract state to $\langle$ OpenRight(0), Int32〉.

Re-execution is justified by Tarski's fixpoint theorem [29, which states that in a partial order $\operatorname{Ifp}(F)=\sqcap\{I \mid F(I) \sqsubseteq I\}$. So, if $I$ is a post-fixpoint for $F$, then $F(I)$ is still above the least fixpoint $\operatorname{Ifp}(F)$, and hence it is a sound approximation of the loop invariant. During re-execution, we refine the abstract semantics of the tests appearing in loops which involve inequalities where one of the operands is an Int32. For instance in the loopToN example:

$$
\mathbb{N} \llbracket \mathrm{x}<\mathrm{n} \rrbracket([\mathrm{x} \mapsto\langle\text { OpenRight }(0), \text { Float64 }\rangle])=\left[\mathrm{x} \mapsto\left\langle\operatorname{Normal}\left(0,2^{31}-2\right), \text { Int } 32\right\rangle\right] .
$$

In Ex. 7 we pointed out that in general it is not sound to assume $\mathrm{x}$ : Int32 after a test $\mathrm{x}<\mathrm{y}$ when $\mathrm{y}$ : Int32. However during re-execution this is sound as there are essentially three cases why $\mathrm{x}$ : Float64 in $I$ : (i) x was a Float64 at the loop entry; (ii) x may be assigned a fractional value (or NaN or an infinite) in the loop body; or (iii) the analysis of the loop could not figure out that $\mathrm{x}$ : Int32. In the first two cases, $F(I)$ will imply that $\mathrm{x}$ : Float64 (because of the definition of $F)$. In the third case one may hope to recover some of the lost precision. In our running example:

$$
\begin{aligned}
F(I) & =[\mathrm{x} \mapsto\langle\operatorname{Normal}(0,0), \operatorname{Int} 32\rangle] \dot{ப}_{k i}\left[\mathrm{x} \mapsto\left\langle\operatorname{Normal}\left(1,2^{31}-1\right), \operatorname{Int} 32\right\rangle\right] \\
& =\left[\mathrm{x} \mapsto\left\langle\operatorname{Normal}\left(0,2^{31}-1\right), \operatorname{Int} 32\right\rangle\right] \dot{\bigsqcup}_{k i} I .
\end{aligned}
$$

$\left(\right.$ Recall that $\gamma_{k i}\left(\left\langle\operatorname{Normal}\left(0,2^{31}-1\right), \operatorname{Int} 32\right\rangle\right)=\gamma_{k i}(\langle$ OpenRight $\left.(0), \operatorname{Int} 32\rangle)\right)$.

Ignored Statements and Havoc. Ignored statements have no effect on the local state, so the analysis treats them as the identity : $\mathbb{N} \llbracket \operatorname{Ignored}_{\mathrm{C}} \rrbracket\left(\sigma_{0}\right)=\sigma_{0}$. Havoc statements may have some side-effect on local variables. We abstract them by $\mathbb{N} \llbracket \operatorname{Havoc}_{\mathbb{C}} \rrbracket\left(\sigma_{0}\right)=\left\langle\top_{i}, \top_{k}\right\rangle$.

\subsection{Variation Analysis}

The numerical analysis alone cannot determine that $c$ : Int32 in bitsinbyte (Fig. 11). It discovers the loop invariant $\sigma_{L}=[\mathrm{m} \mapsto\langle\operatorname{Normal}(1,512)$, Int 32 $\rangle, \mathrm{c} \mapsto$ 〈OpenRight(0),Float64)] (we omit b). The invariant $\sigma_{L}$ can be refined by the variation analysis. At the loop entry, $c$ and $m$ differ by one. At each iteration $c$ is either incremented by one or it remains the same, whereas $\mathrm{m}$ is multiplied by 2 , thus $\mathrm{m}$ grows faster than $\mathrm{c}$. However, $\mathrm{m}$ bounded implies that $\mathrm{c}$ should be bounded too, thus we can safely refine $\sigma_{L}$ to $\sigma_{L}[\mathrm{c} \mapsto\langle\operatorname{Normal}(0,512)$, Int32 $\rangle]$. 
We run the variation analysis $\mathbb{V} \llbracket \cdot \rrbracket$ on a per-loop basis. The goal of the analysis is to compute, for each loop and each variable an interval over-approximating the increment of a variable in a single loop iteration. The variation analysis is similar in many aspects to the numerical analysis above, with the major difference that the initialization and the assignments are re-interpreted. An abstract state is a map from local variables to intervals. At the loop entry point, all the local variables are set to the interval $[0,0] 2$ (no increment). For assignments, we compute variable increments. We consider simple forms of increments and decrements, and we abstract away all the other expressions. So, we let $\mathbb{V} \llbracket \mathrm{x}=\mathrm{x} \pm k \rrbracket\left(\sigma_{0}\right)=\sigma_{0}[\mathrm{x} \mapsto \pm[k, k]]$, and $\mathbb{V} \llbracket \mathrm{x}=\mathrm{e} \rrbracket\left(\sigma_{0}\right)=\sigma_{0}[\mathrm{x} \mapsto[-\infty,+\infty]]$.

Once we have computed $\nu$, the increment ranges for the variables in the loop, we use this information to refine the numerical loop invariant $\sigma_{L}$ to $\sigma_{L}^{\prime}$ according to refinement rules that looks like:

$$
\begin{gathered}
\forall \mathrm{x}, \mathrm{y} \cdot \mathrm{x} \neq \mathrm{y} \wedge \sigma_{0} \models \mathrm{x}<\mathrm{y} \wedge \mathrm{y} \text { is upper-bounded by } b \wedge \nu \models \mathrm{x}<\mathrm{y} \\
\Longrightarrow \sigma^{\prime}{ }_{L}(\mathrm{x})=\sigma_{L}(\mathrm{x}) \dot{\Pi}_{k i}\left\langle\text { OpenLeft }(b), \top_{k}\right\rangle,
\end{gathered}
$$

(the intuitive meaning of $\sigma_{0} \models \mathrm{x}<\mathrm{y}$ is that in the k-interval $\sigma_{0}, \mathrm{x}<\mathrm{y}$ and the meaning of $\nu=\mathrm{x}<\mathrm{y}$ is that according to $\nu, \mathrm{x}$ grows slower than $\mathrm{y})$. The rule above essentially states that if $\mathrm{y}$ is an upper bound for $\mathrm{x}$ at the entry of the loop, and $\mathrm{y}$ is bounded by $b$ during all the executions of the loop, and $\mathrm{x}$ does not grow more than $\mathrm{y}$ in the loop, then $b$ should be an upper bound for $\mathrm{x}$ too. We omit all the other (tedious) refinement rules, which consider the combination of the other cases (e.g., $\sigma_{0} \models \mathrm{x} \leq \mathrm{y}$, lower bounds, decrements and so on).

\subsection{Atomic Types}

The atomic types $T$ for a function are obtained by joining together the poststates of all the statements in the function body. The reason for that is that we want to assign a unique atomic type at each local variable. One may wonder why we designed a flow-sensitive analysis if we were interested in a flow-insensitive property (the type of a local variable through all the function's body). Actually, in an early stage of this project we tried to avoid the joining phase by designing a flow-insensitive analysis. For instance, the abstract semantics of the sequence was $\mathbb{N} \llbracket \mathbf{C}_{1} C_{2} \rrbracket\left(\sigma_{0}\right)=$ let $\sigma=\mathbb{N} \llbracket \mathbf{C}_{1} \rrbracket\left(\sigma_{0}\right)$ in $\mathbb{N} \llbracket \mathbf{C}_{2} \rrbracket(\sigma) \dot{ப}_{k i} \sigma$. We immediately realized that a flow-insensitive analysis was too imprecise for handling loops, and in particular it voided the advantages of the re-execution step and the variation analysis which we found crucial for precision. Therefore, we rejected the flowinsensitive analysis for a flow-sensitive followed by a join-all step.

\section{Experiments}

We have implemented RATA in our JavaScript engine for .Net. The engine itself is written in $\mathrm{C \#}$. It parses the JavaScript source, it compiles the main

\footnotetext{
${ }^{2}$ We use the notation $[a, b]$ to avoid confusion between the range intervals of the previous sections and the increment intervals. In the implementation we share the code, though.
} 


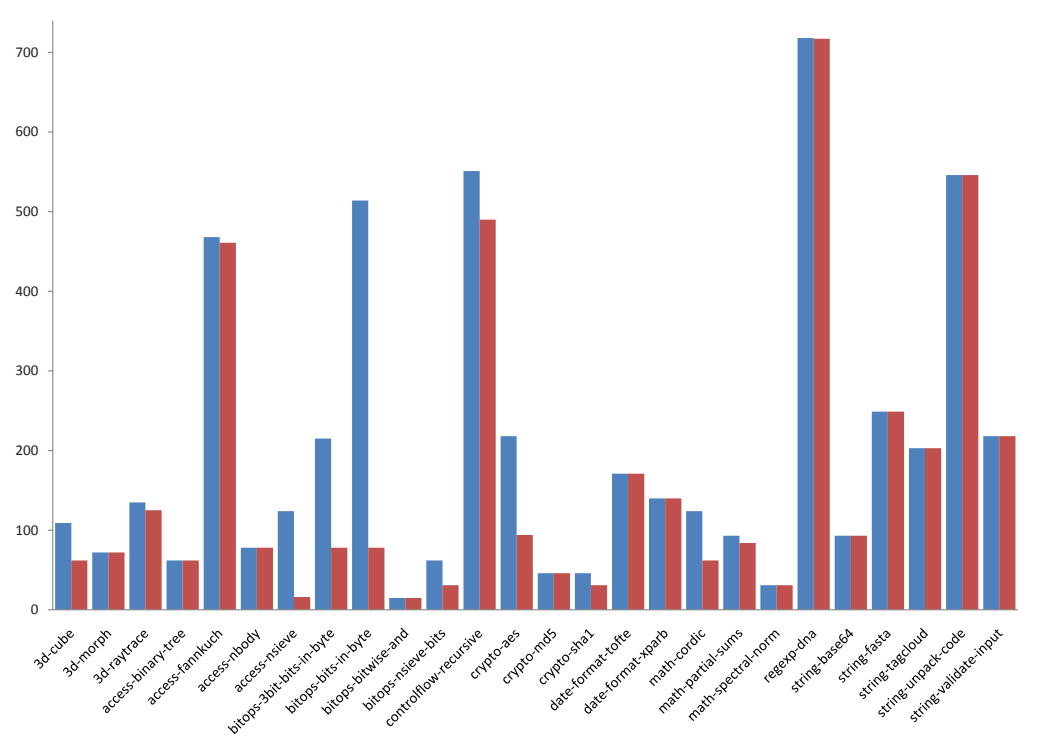

Fig. 7. The results of the optimizations enabled by a text-book type inference algorithm (blue/light bars) and RATA (red/dark bars). Times are expressed in milliseconds. On numerical intensive benchmarks RATA enables up to a $7.7 \times$ speed-up.

(global) function and it generates proxies for function invocations. When the execution encounters a function proxy, the JavaScript engine resolves it, and it checks if it has a specialized version in the cache which matches the actual parameters. If this is the case, then it executes the cached version. Otherwise: (i) it runs the RATA to infer the atomic types for the locals of the variables; (ii) it compiles the function in memory, performing atomic type specialization; (iii) executes the specialized function, and caches it for future needs. It is worth noting that the specialization is polymorphic: If the same function is invoked at two points of time with two actual parameters of different types, then it is analyzed and specialized twice.

We report the experience of applying RATA on the SunSpider JavaScript benchmarks 31]. The SunSpider benchmarks measure JavaScript performance for problems that presents difficulties to JavaScript implementations. They are designed to be balanced and to stress different areas of the language. They are commonly used to compare the JavaScript performance of different browsers, or different versions of the same browser. We run the experiments on a $2.1 \mathrm{GHz}$ Centrino Duo Laptop, 4Gbyte, under Windows 7 and .Net v3.5. We compared a text-book type inference algorithm [2] with RATA. The type inference algorithm determines which locals are definitely doubles, and for some expressions it can also infer that a local is an Int32.

The results of our experiments are in Fig. 7. Measuring the performances of managed programs is quite complex, as their runtime behavior depends on too many variables [18. In general, when the execution time is too low, it is 
foo may change the value of $x$. Functions are analyzed top-down: first the JIT runs RATA on the global statement, and then, at the first concrete occurrence, it invokes RATA on foo. As a consequence when inferring the type of $\mathrm{x}$, RATA assumes the worst case for foo. Determining $\mathrm{x}$ : Int32 requires a bottom-up purity analysis or an effect analysis [4, which are out-of-the scope of the paper, and in general too expensive to be performed online.

In the access-nsieve benchmark, RATA local inference enables a significant speedup $(7.7 \times)$ over the Float64-specialized version. In particular, the inner function contains two nested loops and a counter variable. Fixpoint computation with re-execution and variation analysis are cardinal to infer that all the locals involved are indeed Int32. The other two benchmarks of the access family benchmarks perform computations which either depend on globals or on very short loops.

The controlflow-recursive benchmark stresses JavaScript implementations with standard recursive-function benckmarks such as fibonacci or ackerman. RATA infers that the variables inside those functions are Int32 and thus achieves a slight performance improvement $(1.12 \times)$.

The cryptographic benchmarks benefit by an aggressive type specialization. RATA infers all the Int32 locals for the crypto-aes and the crypto-sha1 benchmarks, enabling a $2.3 \times$ and $1.5 \times$ speedup. The crypto-md5 benchmark contains many functions taking an array as parameter, and iterating over its elements. The next example shows that it would be unsound to infer those locals to be Int32.

Example 11. Let us consider the zeroarray function of Fig. 8. In JavaScript, the length property of Array is a UInt32, i.e., it can assume values as large as $2^{32}-1$. As a consequence, even if we know that arr is an array, we cannot conclude $\mathrm{x}$ : Int32. In general, to infer that $\mathrm{x}$ : Int32, we should refine RATA to track that arr is an array and that arr. length $<2^{31}-1$. The JavaScript languages allows the redefinition of Array, so we need a global analysis to guarantee that the value of a is actually an array.

The execution time of date and string manipulating benchmarks is heavily dominated by the interaction with the object model, and by other non-numerical computations so that RATA is of no help here.

Math benchmarks manipulate double values, but the inference of some Int32 locals enable up to a $2 \times$ speedup in math-cordic, a slight improvement in math-partialsums. For atomic type inference, the test math-spectral-norm looks like crypto-md5, and as a consequence nothing can be statically inferred.

To sum up, RATA is precise enough to infer all (but 3) of the local variables which are Int32 in the SunSpider benchmarks. One may wonder if broadening the analysis to also consider Int64, UInt32 and so on may provide further performance gains. According to the previous experience of the second author with JScript.NET, those cases are so rare, and they complicate so much the implementation and the JIT code generation, that it seems not worthwhile to try. 


\section{Related Work}

Just-in-time compilation is known at least from 1960. In his LISP paper [25, McCarthy sketches the dynamic compilation of functions into machine code, a process fast enough that the compiler's output does not need to be saved. Deutsch and Schiffman introduced in 14 lazy JIT compilation for Smalltalk, where functions were compiled at the first usage, and cached for further usage. The Self programming language influenced the JavaScript design. The first Self compiler used a data-flow analysis ("Class analysis") to compute an overapproximation of the set of possible classes that variables might hold instances of and hence to optimize dynamic dispatching [8]. Further versions of the Self compiler introduced more aggressive type analyses [30, but they did not consider the specialization of atomic types as here [1.

The implementation of popular dynamic languages as Python try to optimize the generated code by performing some kind of online static analysis. The JIT compiler of the PyPy system 28] uses "flexswitches" to perform type specialization [13. Flexswitches are essentially a form of online partial evaluation [21]. Psyco [27] is another implementation of Python which tries to guess Int32 variables at runtime. The tracing JIT generalizes the ideas of Psyco and PyPy. A tracing JIT essentially identifies frequently executed loop traces at runtime, and it dynamically generates specialized machine code [17]. RATA is complementary to a tracing JIT. In his master thesis, Cannon presented a localized atomic type inference algorithm for Python [7. His analysis is based on the Cartesian product algorithm, and it is less precise than ours. As a consequence, it is not a surprise that his experimental results are less satisfactory than ours. In [3], Anderson et al. introduced an algorithm for type inference of JavaScript to derive the types of objects. It is unclear if their algorithm is fast enough to be used in dynamic compilation. They did not consider the inference of Int32 variables which require reasoning on the values of variables. In this sense, our work is then complementary to theirs. In 20, Jensen et al. presented an abstract interpretation based static analysis to check the absence of common errors in JavaScript programs. Their analysis is more oriented to program verification than optimization. However, for numerical values their abstract domain is less precise than ours and so they are not likely to discover all the numerical properties that RATA can discover.

Abstract Interpretation is mainly applied to program verification (e.g., 6]15) and offline program optimization (e.g., 10/5). To the best of our knowledge this is the first work which applies full-powered Abstract Interpretation techniques (e.g., infinite lattices, widenings and narrowings) to online program optimization. We believe that this is a promising line of work.

\section{Conclusions}

We have presented RATA, a new static analysis, based on abstract interpretation, for the rapid inference of atomic types in dynamic languages. The analysis is 
a combination of three analyses: a range analysis, a kind analysis and a variation analysis. We formalized the underlying abstract domains and we related them to the concrete values via Galois connections. We described the analysis, and we reported the results of the atomic type specialization on the SunSpider JavaScript benchmarks (the industrial standard for comparing JavaScript implementations). We observed that: (i) RATA is precise enough to infer all the Int32 locals that one may hope to infer statically; and (ii) the Int32-specialization produces remarkable performance improvements in most tests (up to a $7.7 \times$ speed-up for numerical intensive ones).

For the future, we plan to extend RATA to whole program analysis, and in particular to apply it to the wider goal of program verification.

\section{References}

1. Agesen, O., Hölzle, U.: Type feedback vs. concrete type inference: A comparison of optimization techniques for object-oriented languages. In: OOPSLA 1995. ACM Press, New York (1995)

2. Aho, A.V., Sethi, R., Ullman, J.D.: Compilers: Principles, Techniques, and Tools. Addison-Wesley, Reading (1986)

3. Anderson, C., Giannini, P., Drossopoulou, S.: Towards type inference for javascript. In: Black, A.P. (ed.) ECOOP 2005. LNCS, vol. 3586, pp. 428-452. Springer, Heidelberg (2005)

4. Barnett, M., Fähndrich, M., Garbervetsky, D., Logozzo, F.: Annotations for (more) precise points-to analysis. In: IWACO 2007 (2007)

5. Blanchet, B.: Escape Analysis: Correctness proof, implementation and experimental results. In: POPL 1998 (1998)

6. Blanchet, B., Cousot, P., Cousot, R., Feret, J., Mauborgne, L., Miné, A., Monniaux, D., Rival, X.: A static analyzer for large safety-critical software. In: PLDI 2003. ACM Press, New York (2003)

7. Cannon, B.: Localized type inference of atomic types in Python. Master's thesis, California Polytechnic State University (2005)

8. Chambers, C., Ungar, D.: Customization: Optimizing compiler technology for self, a dynamically-typed object-oriented programming language. In: PLDI 1989. ACM Press, New York (1989)

9. Cousot, P.: The calculational design of a generic abstract interpreter. In: Calculational System Design. NATO ASI Series F. IOS Press, Amsterdam (1999)

10. Cousot, P., Cousot, R.: Abstract interpretation: a unified lattice model for static analysis of programs by construction or approximation of fixpoints. In: POPL 1977 (1977)

11. Cousot, P., Cousot, R.: Systematic design of program analysis frameworks. In: POPL 1979 (1979)

12. Cousot, P., Halbwachs, N.: Automatic discovery of linear restraints among variables of a program. In: POPL 1978 (1978)

13. Cuni, A., Ancona, D., Rigo, A.: Faster than C\#: Efficient implementation of dynamic languages on.NET. In: ICOOOLPS 2009. ACM Press, New York (2009)

14. Deutsch, L.P., Schiffman, A.M.: Efficient implementation of the smalltalk- 80 system. In: POPL1980. ACM Press, New York (1980)

15. Ferrara, P., Logozzo, F., Fähndrich, M.A.: Safer unsafe code in.Net. In: OOPSLA 2008 (2008) 
16. Flanagan, D.: JavaScript, the definitive guide. O'Reilly, Sebastopol (2009)

17. Gal, A., Eich, B., Shaver, M., Anderson, D., Mandelin, D., Haghighat, M., Kaplan, B., Hoare, G., Zbarsky, B., Orendorff, J., Ruderman, J., Smith, E., Reitmaier, R., Bebenita, M., Chang, M., Franz, M.: Trace-based just-in-time type specialization for dynamic languages. In: PLDI 2009 (2009)

18. Georges, A., Eeckhout, L., Buytaert, D.: Java performance evaluation through rigorous replay compilation. In: OOPSLA 2008 (2008)

19. IEEE. IEEE standard for floating-point arithmetic. Technical report, IEEE (2008)

20. Jensen, S.H., Møller, A., Thiemann, P.: Type analysis for javascript. In: Palsberg, J., Su, Z. (eds.) SAS 2009. LNCS, vol. 5673, pp. 238-255. Springer, Heidelberg (2009)

21. Jones, N.D., Gomard, C.K., Sestoft, P.: Partial evaluation and automatic program generation. Prentice-Hall, Englewood Cliffs (1993)

22. Laviron, V., Logozzo, F.: Refining abstract interpretation-based static analyses with hints. In: Hu, Z. (ed.) APLAS 2009. LNCS, vol. 5904, pp. 343-358. Springer, Heidelberg (2009)

23. Laviron, V., Logozzo, F.: Subpolyhedra: a (more) scalable approach to infer linear inequalities. In: Jones, N.D., Müller-Olm, M. (eds.) VMCAI 2009. LNCS, vol. 5403, pp. 229-244. Springer, Heidelberg (2009)

24. Logozzo, F., Fähndrich, M.A.: Pentagons: A weakly relational abstract domain for the efficient validation of array accesses. In: SAC 2008 (2008)

25. McCarthy, J.: Recursive functions of symbolic expressions and their computation by machine, part i. Commun. ACM 3(4), 184-195 (1960)

26. Miné, A.: A new numerical abstract domain based on difference-bound matrices. In: Danvy, O., Filinski, A. (eds.) PADO 2001. LNCS, vol. 2053, p. 155. Springer, Heidelberg (2001)

27. Rigo, A.: Representation-based just-in-time specialization and the psyco prototype for Python. In: PEPM 2004. ACM Press, New York (2004)

28. Rigo, A., Pedroni, S.: PyPy's approach to virtual machine construction. In: OOPSLA Companion 2006. ACM Press, New York (2006)

29. Tarski, A.: A lattice-theoretical fixpoint theorem and its applications. Pacific Journal of Mathematics 5, 285-309 (1955)

30. Ungar, D., Smith, R.B., Chambers, C., Hölzle, U.: Object, message, and performance: How they coexist in self. IEEE Computer 25(10), 53-64 (1992)

31. WebKit. SunSpider JavaScript benchmarks, http://www2.webkit.org/perf/sunspider-0.9/sunspider.html 